\title{
Phosphatidylserine transport by ORP/Osh proteins is driven by phosphatidylinositol 4-phosphate
}

\begin{abstract}
Joachim Moser von Filseck, ${ }^{1 *}$ Alenka Čopič, ${ }^{2 *}$ Vanessa Delfosse, ${ }^{3,4 *}$ Stefano Vanni, ${ }^{1}$ Catherine L. Jackson, ${ }^{2}$ William Bourguet, ${ }^{3,4}$ Guillaume Drin ${ }^{1} \dagger$

In eukaryotic cells, phosphatidylserine (PS) is synthesized in the endoplasmic reticulum (ER) but is highly enriched in the plasma membrane (PM), where it contributes negative charge and to specific recruitment of signaling proteins. This distribution relies on transport mechanisms whose nature remains elusive. Here, we found that the PS transporter Osh6p extracted phosphatidylinositol 4-phosphate (PI4P) and exchanged PS for PI4P between two membranes. We solved the crystal structure of Osh6p:PI4P complex and demonstrated that the transport of PS by Osh6p depends on PI4P recognition in vivo. Finally, we showed that the PI4P-phosphatase Saclp, by maintaining a PI4P gradient at the ER/PM interface, drove PS transport. Thus, PS transport by oxysterol-binding protein-related protein (ORP)/oxysterol-binding homology (Osh) proteins is fueled by PI4P metabolism through PS/PI4P exchange cycles.
\end{abstract}

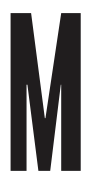

embranes of eukaryotic cells vary in their lipid composition, a feature essential to organelle identity $(1,2)$. It is unclear how lipids are conveyed across the cytosol or at organelle junctions and precisely distributed in the cell (3-5). The plasma membrane (PM) is highly enriched in the anionic phospholipid phosphatidylserine (PS), which recruits and activates various signaling proteins (6). How PS, synthesized in the endoplasmic reticulum (ER)

Institut de Pharmacologie Moléculaire et Cellulaire, Université de Nice Sophia-Antipolis and CNRS, 660 route des lucioles, 06560 Valbonne, France. ${ }^{2}$ Institut Jacques Monod, CNRS, UMR 7592, Université Paris Diderot, Sorbonne Paris Cité, F-75013 Paris, France. Inserm U1054, 29 rue de Navacelles, 34090 Montpellier, France. ${ }^{4}$ CNRS UMR5048, Centre de Biochimie Structurale, 29 rue de Navacelles, 34090 Montpellier, France.

*These authors contributed equally to this work. †Corresponding author. E-mail: drin@ipmc.cnrs.fr
$(7,8)$, reaches its final destination remains unclear. In yeast, PS is transferred from the ER to the PM by Osh6p or Osh7p (9), two members of the oxysterol-binding protein (OSBP)-related protein (ORP)/oxysterol-binding homology (Osh) family (10). What drives this directional process is unknown.

Osh6p and Osh7p contain a lipid-binding domain-called ORD (OSBP-related domain)for transferring PS (9), with a short upstream region (fig. $\mathrm{S} 1$ ). Other $\mathrm{ORP} / \mathrm{Osh}$ proteins have an ORD specific for sterol (10-12), and two of them, OSBP and Osh4p, exchange sterol with a second lipid, phosphatidylinositol 4-phosphate (PI4P) $(13,14)$. Like other phosphoinositides, PI4P is confined to specific organelles via the local activity of PI kinases and phosphatases (15). PI4P levels are higher in the trans-Golgi and PM than in the ER. OSBP and Osh4p, by sterol/PI4P exchange, use this PI4P gradient to ensure ER-to- 
Golgi sterol transport $(14,16)$. Presumably, PI4P is a ligand for all ORP/Osh proteins $(13,17)$. We thus looked for a link between PI4P and Osh6p/ Osh7p transport function.

First, we investigated whether purified Osh6p/ Osh7p extracted PI4P from model membranes. We used a PI4P sensor, NBD-PH FAPP $_{\text {(PH domain }}$ of the four-phosphate-adaptor protein 1) (16), bearing a polarity-sensitive (7-nitrobenz-2-oxa-1,3-diazol) NBD fluorophore, whose emission intensity depends on whether it is soluble or membrane-bound. In the absence of Osh proteins, NBD-PH $\mathrm{FAPP}_{\mathrm{FAs}}$ fully bound to PI4P-containing liposomes. Adding Osh6p or Osh7p resulted in a decrease in fluorescence, indicating that they extracted PI4P from liposome ( 80 to $95 \%$ of accessible PI4P) (Fig. 1A). In control experiments, wild-type (WT) Osh4p removed PI4P, whereas Osh4p(H143A/ H144A), deficient in binding PI4P (16), did not. Unlike Osh4p, Osh6p and Osh7p did not extract dehydroergosterol (DHE), a fluorescent ergosterol (fig. S2).

Consequently, we hypothesized that Osh6p/ Osh7p might be lipid exchangers like Osh4p but specific for PS and PI4P. Because Osh6p and Osh7p are close homologs, we focused the rest of our study on Osh6p. With the PI4P extraction assays, we first investigated whether PS competes with PI4P for binding Osh6p using lipids (16:0/18:1 PS and 16:0/16:0 PI4P) that most closely resemble the major yeast PS and PI4P species (18). When liposomes contained PI4P and a 2.5-fold excess of PS, the extraction of PI4P by Osh6p was inhibited (Fig. 1B). DHE at the same concentration did not block extraction. Conversely, DHE, but not PS, prevented Osh4p from extracting PI4P. Thus, Osh6p binds PS and PI4P specifically in a mutually exclusive manner.

Fig. 1. Osh6p is a PS/PI4P exchanger. (A) Fluores cence spectra of NBD-PH FAPP $(250 \mathrm{nM})$ mixed with liposomes (150 $\mu \mathrm{M}$ lipid, 4\% PI4P) in the absence or presence of Osh protein $(3 \mu \mathrm{M})$. Normalized signal gives the percentage of extracted PI4P. (B) PI4P extraction by Osh protein from liposomes containing $4 \%$ PI4P and, additionally, $10 \%$ PS or DHE. (C) PS transport by Osh protein $(200 \mathrm{nM})$ injected into a suspension of $\mathrm{L}_{\mathrm{A}}$ liposomes containing 5\% PS and $2 \%$ Rhod-PE, mixed with NBD-C2 Lact $(250 \mathrm{nM})$ and $\mathrm{L}_{\mathrm{B}}$ liposomes (with 0 or $4 \%$ PI4P). (D) PI4P transport by Osh protein added to $L_{B}$ liposomes containing 4\% PI4P and 2\% Rhod-PE, mixed with NBD-PH FAPP $(250 \mathrm{nM})$ and $\mathrm{L}_{\mathrm{A}}$ liposomes (with or without $P S)$. $\left[L_{A}\right]=\left[L_{B}\right]=200 \mu \mathrm{M}$ total lipids. NBD signals were converted into PS and PI4P amount $(\mu \mathrm{M})$ in $L_{B}$ and $L_{A}$ liposomes, respectively. Dashed line, equilibrium. All values are means $\pm \operatorname{SEM}(N=3$ experiments).
Next, we examined whether Osh6p exchanged PS with PI4P between membranes. To measure PS transport, we created a fluorescent sensor (NBD-C2 $\left.{ }_{\text {Lact }}\right)$, based on the lactadherin $\mathrm{C} 2$ domain (19), selective for PS (fig. S3). NBD-C2 $2_{\text {Lact }}$ was mixed with two liposome populations, one $\left(\mathrm{L}_{\mathrm{A}}\right)$ containing $5 \%$ PS and $2 \%$ rhodaminephosphatidylethanolamine (Rhod-PE) and the other $\left(\mathrm{L}_{\mathrm{B}}\right)$ only made of phosphatidylcholine. The signal of NBD-C2 $2_{\text {Lact }}$, bound to $\mathrm{L}_{\mathrm{A}}$ liposomes, was quenched due to fluorescence resonance energy transfer with Rhod-PE. Adding Osh6p provoked dequenching because it transported PS to $\mathrm{L}_{\mathrm{B}}$ liposomes, thereby promoting the translocation of NBD-C2 $2_{\text {Lact }}$ (Fig. 1C). The initial transport rate (calculated from the signal converted into PS amount in $\mathrm{L}_{\mathrm{B}}$ liposomes) was $0.9 \pm 0.7 \mathrm{PS} / \mathrm{min}$ per Osh6p (mean \pm SEM, $N=3$ experiments). Osh $4 p$ was almost inactive $(0.14 \pm 0.02 \mathrm{PS} / \mathrm{min})$. When $\mathrm{L}_{\mathrm{B}}$ liposomes contained PI4P, Osh6p transported PS 10 times faster $(15.4 \pm 1.5 \mathrm{PS} / \mathrm{min})$, whereas only a slight increase was seen with Osh4p (Fig. 1C). Osh6p moved short and saturated PS species faster (fig. S4).

Using a similar assay with NBD-PH $\mathrm{PAPP}_{\mathrm{FP}}$, we followed PI4P transport in the back direction, from $\mathrm{L}_{\mathrm{B}}$ liposomes with $4 \%$ PI4P and $2 \%$ Rhod-PE to $\mathrm{L}_{\mathrm{A}}$ liposomes (containing or not containing $5 \% \mathrm{PS}$ ). In the absence of PS, Osh6p transferred PI4P at an initial velocity of 8 PI4P/min (Fig. 1D). Transport increased by a factor of about 2 in the presence of PS. In control assays, PI4P transport by Osh4p was slightly enhanced by PS but was much faster when $\mathrm{L}_{\mathrm{A}}$ liposomes contained the proper counterligand DHE. Thus, Osh6p transported PS and PI4P along opposite routes. Both lipids were transported faster, with similar velocities, when they were initially in distinct membranes, suggesting that Osh6p is a PS/PI4P exchanger

Next, we solved the crystal structure of Osh6p in complex with PI4P to define how it binds this lipid (Fig. 2A, fig. S5A, and table S1). The structure (at $2.55 \AA$ resolution) is similar to that of PSbound Osh $6 \mathrm{p}$ (root mean square deviation $=0.36 \AA$ for 403 residues) (fig. S5, B and C). The electron density of ligand corresponds to 18:0/20:4 PI4P (main PI4P species used for crystallization). The stearate (18:0 chain) at the $s n-1$ position of the glycerol backbone of PI4P is deeply inserted into the binding pocket, whereas the $s n$-2 arachidonate (20:4) is twisted, pointing its end to the lid (residues 35 to 69 ) that closes the pocket (Fig. 2A). The 4-phosphate group makes hydrogen bonds with H157, H158, and R359 side chains and water-mediated hydrogen bonds with R66 and E355 main chains. The 1-phosphate group interacts with the backbone amide of L69, K126, N129, and K351. Free hydroxyl groups of inositol interact with T65, N129, and E355. As seen for other ORD structures $(13,17)$, PI4P recognition relies on residues conserved in $\mathrm{ORP} / \mathrm{Osh}$ family members, like the histidine pair, reinforcing the idea that PI4P is a ligand common to all ORP/ Osh proteins.

Molecular dynamics (MD) simulations were carried out to compare how PS or PI4P bind to Osh6p. Both lipids had limited motion in the binding pocket (fig. S6, A and B). The mobile parts of Osh6p (except loops) were the lid and $\alpha 7$ helix. No differences in the amplitude and/or opening of the lid were seen between the complexes (fig. S6C). The interaction energy between PI4P and Osh6p was $\sim 50 \%$ higher $(-1029.6 \mathrm{~kJ} / \mathrm{mol})$ than that between PS and Osh6p $(-628 \mathrm{~kJ} / \mathrm{mol})$. In both cases, the contribution of acyl chains to the
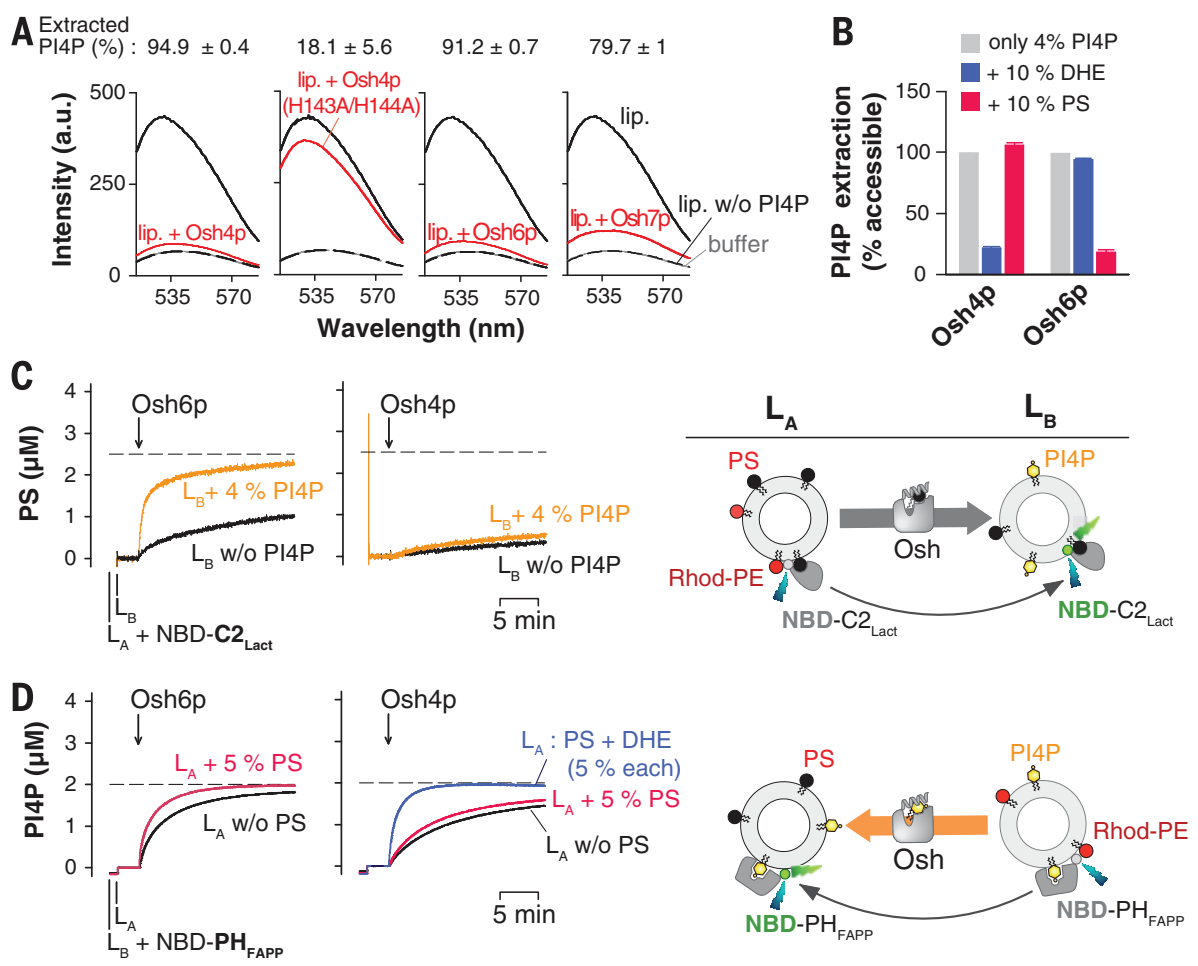
interaction was similar (about $-300 \mathrm{~kJ} / \mathrm{mol}$ ) (Fig. 2B). In contrast, some residues (R66, H157, H158, and R359) made strong and almost exclusive contacts with PI4P (Fig. 2C and fig. S7), accounting for the differential interaction energy calculated for PS and PI4P. The binding of the 4-phosphate group to Osh6p $(-517 \mathrm{~kJ} / \mathrm{mol})$ represented $50 \%$ of the Osh6p-PI4P interaction energy.

Based on our structural analysis, we designed Osh6p mutants deficient in binding PI4P and/or PS: a H157A/H158A mutant lacking the histidines that recognize PI4P, a reverse-charge mutant K351E (K351 binds PI4P and, as suggested by simulation, PS), and a $\Delta 69$-Osh $6 \mathrm{p}$ mutant (residues 70 to 448 ) lacking the $\mathrm{N}$-terminal region and ORD lid. We tested the transport activity of these mutants and of Osh6p(L69D), deficient in binding PS (9). Like Osh6p WT, all mutants slowly trans- ported PS or PI4P in the absence of counterligand, with $\Delta 69$-Osh6p showing no PS transport. In assays with both lipids, all mutants had a slower PS and PI4P transport activity compared with Osh6p WT. Whereas Osh6p(L69D) was defective for both PI4P and PS transport, Osh6p(K351E) and Osh6p(H157A/H158A) did not transfer PI4P while transporting PS at a moderate rate, and $\Delta 69$-Osh $6 \mathrm{p}$ transported some PI4P but no PS (Fig. 2D). The mutants that still transport lipid into acceptor membranes without transporting back the counterligand likely retain some affinity for this latter, allowing them to better target these membranes. Our assays, showing that PS and PI4P transport rates appeared linked and were maximal only when Osh6p could properly recognize both lipids, confirmed that Osh6p is a PS/PI4P exchanger.
These data suggested that the transport of PS mediated by Osh6p at the ER/PM interface might depend on PI4P. To test this hypothesis in vivo, we expressed Osh6p-mCherry WT or mutants in a cho1s strain lacking endogenous Osh6p and Osh7p to compare their PS-transport ability. The chold mutation blocks PS synthesis, greatly diminishing PS levels and leading to cytosolic localization of $\mathrm{C} 2_{\text {Lact }}-\mathrm{GFP}$ (green fluorescent protein) $(20,21)$. Lyso-PS added to chols cells is converted into PS and rapidly transferred from the ER to the PM when Osh6p is expressed (9), as indicated by $\mathrm{C} 22_{\text {Lact }}$-GFP redistribution (Fig. $2 \mathrm{E}$ ). Analysis of $\mathrm{C} 2$ Lact $-\mathrm{GFP}$ fluorescence profiles showed a large increase of peripheral intensity peaks compared with internal intensity peaks (Fig. 2F and fig. S8). In contrast, when Osh6p was missing or replaced by Osh6p(L69D), the
A

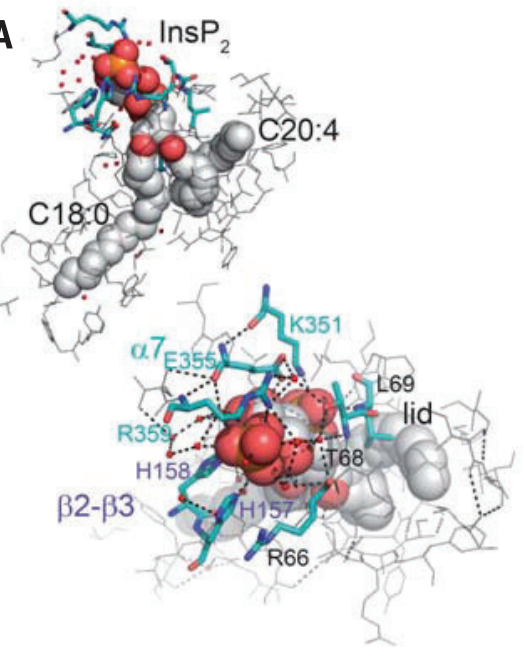

B

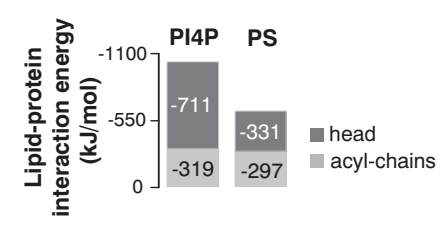

C

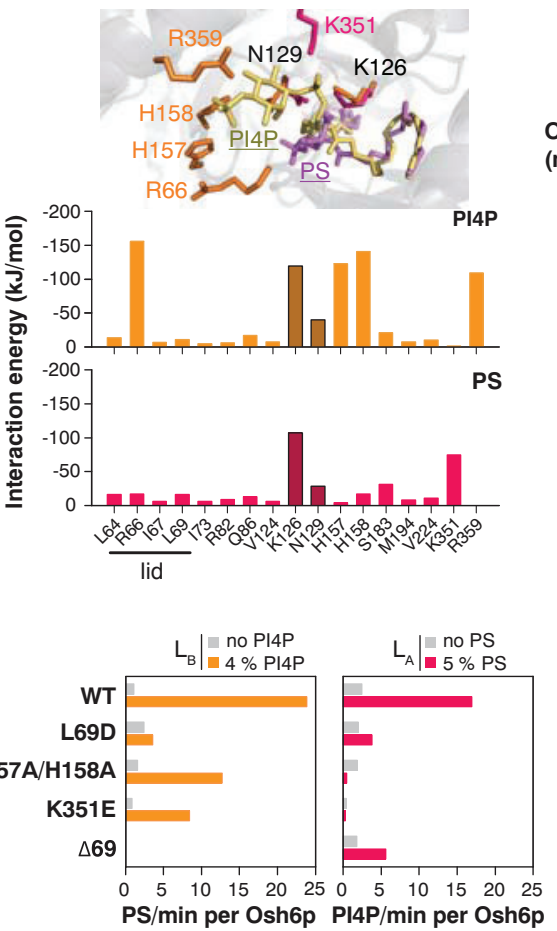

E

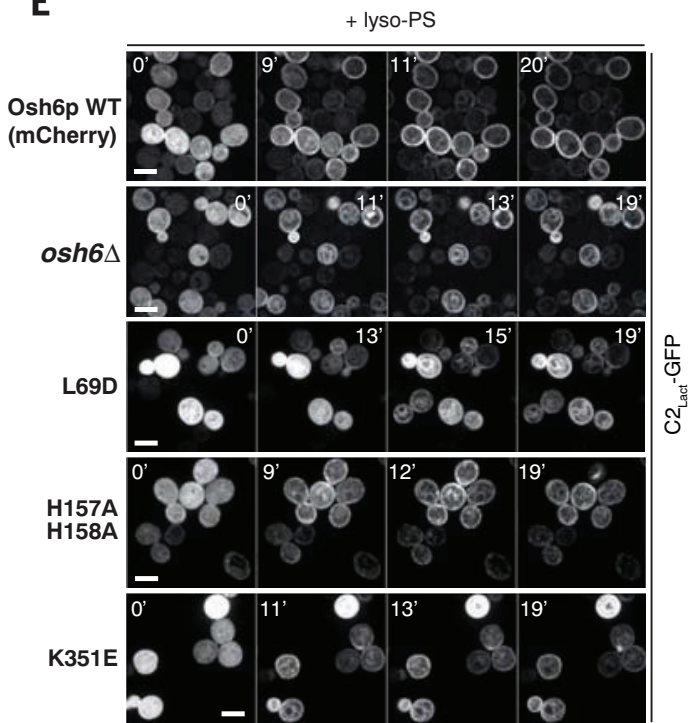

Fig. 2. Cellular Osh6p activity depends on PI4P recognition. (A) Structure of PI4P-bound Osh6p (PI4P in sphere). Closeup view: key residues coordinating the head group. Red dots, water molecules; dashed lines, $\mathrm{H}$ bonds. (B) Interaction energy of 16:0/18:1-PS or 18:0/20:4-PI4P with Osh6p. (C) Main interaction energy between PS or PI4P and Osh6p residues (mean; $N=2$ experiments). K126 and N129 are key interacting residues for both lipids. (D) PS and PI4P transport activity of Osh6p WT or mutants $(200 \mathrm{nM})$ when, initially, the second lipid is absent or the two lipids are present in distinct liposomes (mean; $N=2$ experiments). (E) Redistribution of $C_{2}$ Lact - GFP in PS-depleted yeast cells (osh6 $\Delta$ osh7 $\Delta$ chol $1 \Delta$ ) expressing Osh6p-mCherry WT or mutants after adding 18:1 lyso-PS. Time points: lyso-PS injection ( 0 min), first transition in signal, maximal ER signal, end of time course. Scale bar, $5 \mu \mathrm{m}$. (F) Quantification of C2 Lact-GFP localization showing relative levels in peripheral (red) and internal (blue) membrane signal peaks (mean $\pm \mathrm{SD}, N=3$ experiments, $\sim 20$ cells per experiment). Internal peaks $=$ mostly ER; peripheral $=E R+P M$. Time is adjusted (see fig. S8). (Single-letter abbreviations for the amino acid residues are as follows: A, Ala; C, Cys; D, Asp; E, Glu; F, Phe; G, Gly; H, His; I, lle; K, Lys; L, Leu; M, Met; N, Asn; P, Pro; Q, Gln; R, Arg; S, Ser; T, Thr; V, Val; W, Trp; and Y, Tyr.)

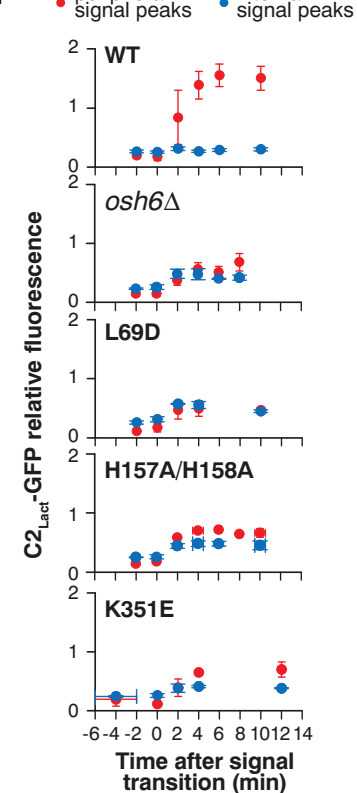


intensities of internal and peripheral signal peaks increased concomitantly, indicating that PS mostly remained at the ER. Compared with Osh6p(L69D), in agreement with our in vitro data, Osh6p(H157A/H158A) and Osh6p(K351E) seemed somewhat less defective in transporting PS to the PM, because the peripheral intensity peaks increased slightly more than the internal peaks (Fig. 2, E and F, and figs. S8 and S9). However, the transport efficiencies were lower than that of Osh6p WT by about an order of magnitude. The mutant Osh6p(H157A/H158A/K351E) displayed a similar phenotype (figs. S8 and S9). Thus, recognizing PI4P is critical for Osh6p to mediate the ER-to-PM PS transport.
To move PS from the ER to the PM, Osh6p should transport PI4P down its gradient in the reverse direction and would be active only if ER PI4P levels remain low. Dephosphorylation of PI4P by the ER-resident Saclp, the major yeast PI4P phosphatase $(22,23)$, should thus be critical for Osh6p activity. PS was mislocalized to internal membranes in $s a c 1 \Delta$ cells (24) (fig. S10A). We probed whether the lack of Saclp affected PS transport directly using a chols sac1s strain and following $\mathrm{C} 22_{\text {Lact }}-\mathrm{GFP}$ redistribution after adding lyso-PS. C2 2 Lact - GFP remained blocked at the ER in sac1 $1 \Delta$ cells, although Osh6p was present (Fig. 3, $\mathrm{A}$ and B, and fig. S10B). The ER-to-PM transport of PS was also blocked in chols sac1-23 cells at
A

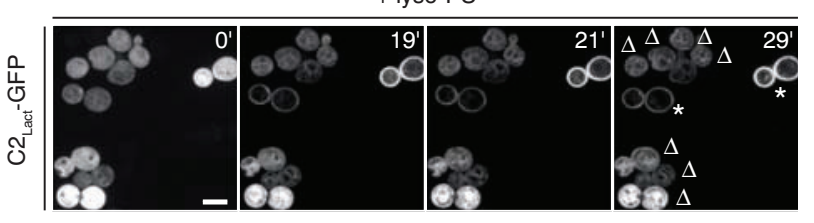

C

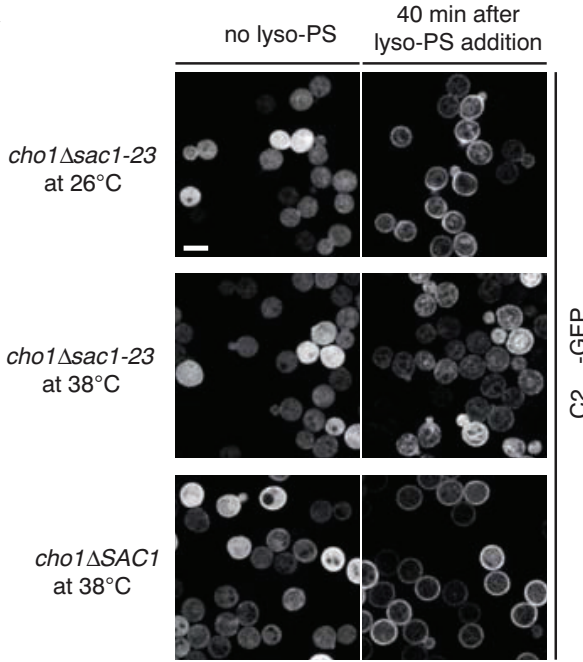

D
B

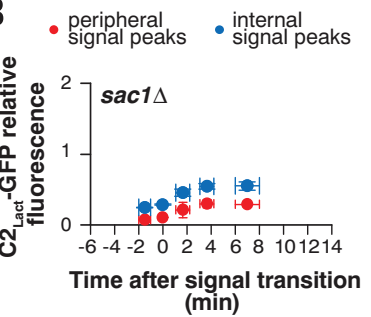

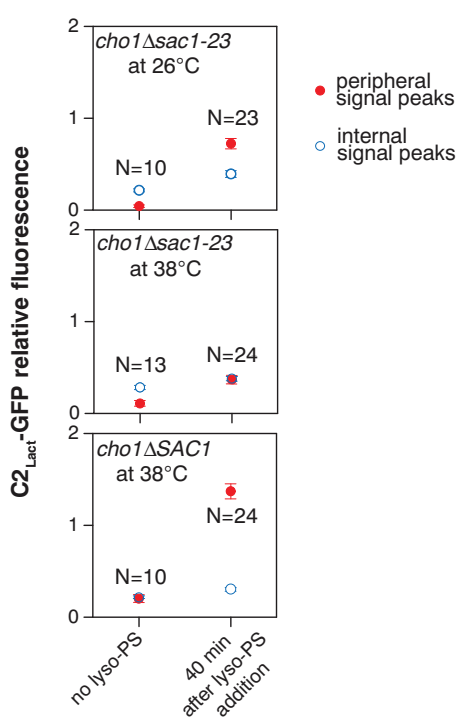

E

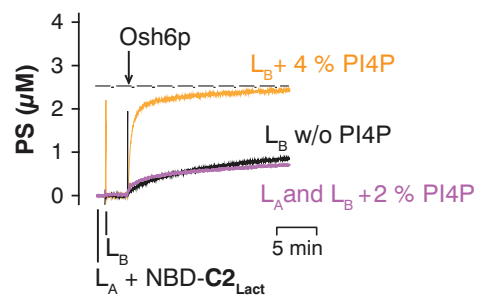

$\mathbf{F}$

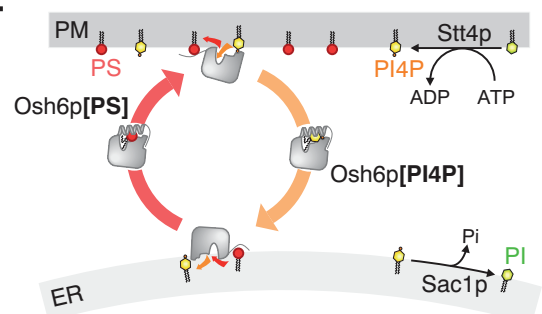

Fig. 3. Sac1p maintains PS transport by Osh6p. (A) C2 Lact-GFP redistribution in $S A C 1\left(^{*}\right)$ and sac1 $\Delta$ cells $(\Delta)$, depleted for PS and expressing Osh6p-mCherry, after adding lyso-PS. Scale bars, $5 \mu \mathrm{m}$. (B)

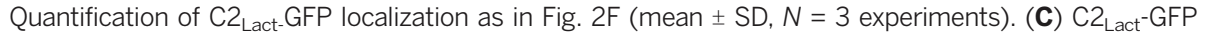
distribution in chol $\Delta$ sacl-23 or chol $1 \Delta$ cells at permissive $\left(26^{\circ} \mathrm{C}\right)$ or nonpermissive temperature $\left(38^{\circ} \mathrm{C}\right)$ for the sacl allele before and after a 40-min incubation with lyso-PS. The experiment was repeated twice; representative images from one experiment are shown. (D) Quantification (mean $\pm \mathrm{SEM}, N=$ number of cells) of the experiments shown in (C). (E) PS transport assays with $L_{B}$ liposomes containing 0 or $4 \%$ PI4P or with $L_{A}$ and $L_{B}$ liposomes, both containing 2\% PI4P. $L_{A}$ liposome contains 5\% PS. (F) Model for the coupling between PI4P metabolism and Osh6p-mediated PS/PI4P exchange.

the nonpermissive temperature (Fig. 3, C and D). In vitro, the PS-transport activity of Osh6p was stimulated when a PI4P gradient preexisted between membranes (as in Fig. 1C) but not when PI4P was equilibrated (Fig. 3E). Saclp might act in trans between the ER and PM (25). We rather propose that it acts in cis, hydrolyzing the PI4P delivered by Osh $6 \mathrm{p}$ and maintaining a PI4P gradient at the ER/PM interface mandatory for its transport activity.

Deciphering the mechanism of lipid transport is key to understanding how organelle identity is maintained (3-5). Osh6p/Osh7p colonize ER-PM junctions, which might contain high PS levels due to concentration of Cholp/Psslp at these sites, allowing PS to be transported along its gradient to the $\operatorname{PM}(9,26)$. Instead, we propose that Osh6p and Osh7p extract PS from the ER, exchange PS with PI4P at the PM, and transport PI4P to the ER. Given the key contribution of the 4-phosphate group for the Osh6p-PI4P interaction, PI4P hydrolysis might facilitate PS uptake from the ER. Maintenance of a PI4P gradient by Saclp (and Stt4p producing PM PI4P) would sustain PS/ PI4P exchange cycles, and thereby PS delivery to the PM, against its concentration gradient (Fig. 3F). These results, along with those obtained with sterol/PI4P exchangers $(13,14,16)$ and ORP5/ ORP8 (27), suggest a general mechanism by which ORP/Osh proteins use PI4P metabolism to build distinct intracellular lipid gradients.

\section{REFERENCES AND NOTES}

1. J. Bigay, B. Antonny, Dev. Cell 23, 886-895 (2012).

2. G. Drin, Annu. Rev. Biochem. 83, 51-77 (2014).

3. W. A. Prinz, Cell 143, 870-874 (2010)

4. T. Levine, Trends Cell Biol. 14, 483-490 (2004)

5. J. C. Holthuis, A. K. Menon, Nature 510, 48-57 (2014).

6. P. A. Leventis, S. Grinstein, Annu. Rev. Biophys. 39 , 407-427 (2010).

7. J. E. Vance, R. Steenbergen, Prog. Lipid Res. 44, 207-234 (2005).

8. E. Zinser et al., J. Bacteriol. 173, 2026-2034 (1991).

9. K. Maeda et al., Nature 501, 257-261 (2013).

10. S. Raychaudhuri, W. A. Prinz, Annu. Rev. Cell Dev. Biol. 26 157-177 (2010)

11. Y. J. Im, S. Raychaudhuri, W. A. Prinz, J. H. Hurley, Nature 437 , 154-158 (2005).

12. N. D. Ridgway, Subcell. Biochem. 51, 159-182 (2010).

13. M. de Saint-Jean et al., J. Cell Biol. 195, 965-978 (2011).

14. B. Mesmin et al., Cell 155, 830-843 (2013).

15. G. Di Paolo, P. De Camilli, Nature 443, 651-657 (2006)

16. J. Moser von Filseck, S. Vanni, B. Mesmin, B. Antonny, G. Drin, Nat. Commun. 6, 6671 (2015).

17. J. Tong, H. Yang, H. Yang, S. H. Eom, Y. J. Im, Structure 21 . 1203-1213 (2013).

18. C. S. Ejsing et al., Proc. Natl. Acad. Sci. U.S.A. 106, 2136-2141 (2009).

19. T. Yeung et al., Science 319, 210-213 (2008)

20. G. D. Fairn et al., J. Cell Biol. 194, 257-275 (2011).

21. W. R. Riekhof et al., J. Biol. Chem. 282, 36853-36861 (2007).

22. M. Foti, A. Audhya, S. D. Emr, Mol. Biol. Cell 12, 2396-2411 (2001).

23. F. Faulhammer et al., Traffic 8, 1554-1567 (2007)

24. M. Tani, O. Kuge, Yeast 31, 145-158 (2014).

25. C. J. Stefan et al., Cell 144, 389-401 (2011)

26. H. Pichler et al., Eur. J. Biochem. 268, 2351-2361 (2001).

27. J. Chung et al., Science 349, 428-432 (2015).

\section{ACKNOWLEDGMENTS}

We thank B. Antonny, M. Dauvois, M. de Saint-Jean, L. d'Esposito,

K. Eudes, C. La Torre, N. Leroudier, S. Léon, the staff of the 


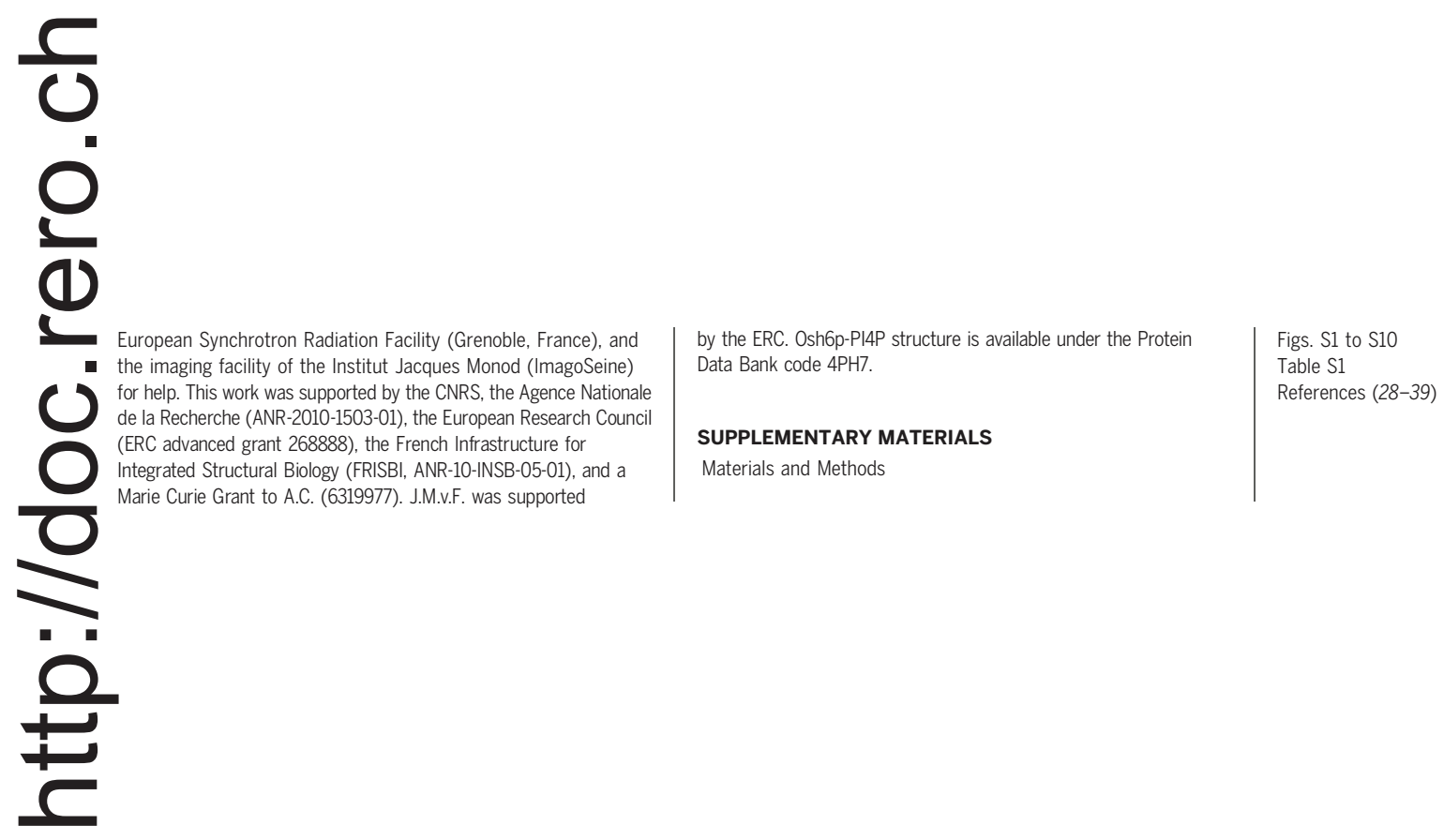

